\title{
THE BRACHISTOCHRONE PROBLEM WITH FRICTIONAL FORCES
}

\author{
Roberto Giambò $^{1}$ And FABio Giannoni ${ }^{2}$
}

\begin{abstract}
In this paper we show the existence of the solution for the classical brachistochrone problem under the action of a conservative field in presence of frictional forces. Assuming that the frictional forces and the potential grow at most linearly, we prove the existence of a minimizer on the travel time between any two given points, whenever the initial velocity is great enough. We also prove the uniqueness of the minimizer whenever the given points are sufficiently close.
\end{abstract}

AMS Subject Classification. 58E30, 58E25, 45J05.

Received October 1, 1999.

\section{INTRODUCTION}

The classical formulation of the brachistochrone problem dates back to 1696, when Johann Bernoulli, from the pages of the review "Acta Eruditorum" directed by Gottfried Leibniz, challenged the mathematicians of the time to find the shortest travel time path of a particle moving between two fixed points in a vertical plane of the space, under the influence of the gravity force.

An earlier formulation had actually been given by Galileo in 1638, although he suggested an arc of circle as the brachistochrone curve. A correct solution of the problem was provided, among the others, by Jakob Bernoulli and Isaac Newton, besides Johann Bernoulli himself, and it is well know today that the curve solution of the problem is an arc of cycloid.

In the generalization that we will study in this paper we consider an arbitrary potential energy $U$, that will be a function on a given manifold $\mathcal{M}$, and a reaction force $R$ of the medium in which the particle moves, depending on the velocity $v$ of the particle itself.

We will denote by $\mathcal{M}$ an $n$-dimensional smooth manifold, $n>1$, endowed with a Riemannian structure $\langle\cdot, \cdot\rangle$. Let us fix the starting and arrival points $p$ and $q$ on $\mathcal{M}$ (we assume $p \neq q$ ), and the initial velocity $v_{0}>0$. For sake of simplicity the particle will be supposed to be of unit mass. The arc length function is given by

$$
\ell(\tau)=\int_{0}^{\tau} \sqrt{\langle\dot{x}(\sigma), \dot{x}(\sigma)\rangle} d \sigma
$$

where $x(\sigma):[0,1] \rightarrow \mathcal{M}$ is the path from $p$ to $q$ expressed in parametric form. Hereafter the dot notation will mean derivative with respect to the parameter.

Keywords and phrases: Minimal travel time, non linear constraints.

1 Dipartimento di Matematica "Ulisse Dini", Università di Firenze, Italy; e-mail: giambo@udini.math.unifi.it

2 Dipartimento di Matematica e Fisica, Università di Camerino, Italy; e-mail: giannoni@campus.unicam.it 
The velocity is given by

$$
v=\frac{d \ell}{d T}
$$

where $T$ is the (absolute) time function that we want to minimize. For our study it will be convenient not to choose $T$ as the parameter; in other words the velocity $v$ will be considered as an "additional" coordinate, in such a way that

$$
\mathbb{R} \times\left(\mathcal{M} \times \mathbb{R}^{+}\right)
$$

will be our configuration space. From (1) and (2) we obtain the functional

$$
T(x, v)=\int_{0}^{1} \frac{\sqrt{\langle\dot{x}(\tau), \dot{x}(\tau)\rangle}}{v(\tau)} d \tau .
$$

Balancing the forces acting along an infinitesimal path $d \ell$ we obtain

$$
\frac{d v}{d T}=-\frac{d U}{d \ell}-R
$$

where $U=U(x)$ is the potential energy and $R=R(v)$ is the frictional force. The left-hand side term can be written as

$$
\frac{d v}{d T}=\frac{d v}{d \ell} \frac{d \ell}{d T}=\frac{d v}{d \tau} \frac{d \tau}{d \ell} v=\frac{\dot{v}}{d \ell / d \tau} v
$$

and then, multiplying both members in (4) by $\frac{d \ell}{d \tau}$ we get

$$
v \dot{v}=-\frac{d U}{d \ell} \frac{d \ell}{d \tau}-R \frac{d \ell}{d \tau}=-\langle D U(x), \dot{x}\rangle-R \sqrt{\langle\dot{x}, \dot{x}\rangle},
$$

that is the constraint equation

$$
\Phi(x, v)=v(s) \dot{v}(s)+\langle D U(x(s)), \dot{x}(s)\rangle+R(v(s)) \sqrt{\langle\dot{x}(s), \dot{x}(s)\rangle}=0
$$

(cf. [5]). Here $D U$ is the gradient of the potential energy,

$$
d U(x)[\xi]=\langle D U(x), \xi\rangle, \quad \forall \xi \in T_{x} \mathcal{M},
$$

and $T_{x} \mathcal{M}$ obviously denotes the tangent space of $\mathcal{M}$ at the point $x \in \mathcal{M}$. Let us observe that integration of (5) yields the conservation of the total energy of the system,

$$
\frac{1}{2} v(s)^{2}+U(x(s))+\int_{0}^{s} R(v(\sigma)) \sqrt{\langle\dot{x}(\sigma), \dot{x}(\sigma)\rangle} d \sigma=\frac{1}{2} v_{0}^{2}+U(p)=E,
$$

where $E$ is the total energy of the particle of unit mass at the initial time. Last addendum in the left-hand side term of (6) is obviously the energy dissipated by the reaction force $R(v)$.

Thus we can formulate the following:

Definition 1.1. Given $p, q \in \mathcal{M}$ and $v_{0}>0$, a (minimal) brachistochrone of energy $E=\frac{1}{2} v_{0}^{2}+U(p)$ from $p$ to $q$ is a couple of curves $(x(s), v(s))$ that minimizes the functional (3) among all the couples of smooth curves 
that

$$
\begin{aligned}
& x:[0,1] \rightarrow \mathcal{M}, x(0)=p, x(1)=q, \\
& v:[0,1] \rightarrow \mathbb{R}^{+}, v(0)=v_{0}, \\
& \Phi(x, v)=0 \text { in }[0,1] .
\end{aligned}
$$

In Theorem 2.1 we prove that, for any couple of points $p$ and $q$ in a complete Riemannian manifold $\mathcal{M}$ there is a brachistochrone joining $p$ and $q$. This is done assuming that the frictional force and the potential have a growth not more than linear ( $c f .(10)$ and (11)). Moreover we obtain the differential equation satisfied by the brachistochrone curves. It is given by (15) where the couple of smooth curves $(x, v)$ satisfies also (14) and the constraint equation (5). To prove Theorem 2.1 one could also try to use the "action functional"

$$
A(x, v)=\int_{0}^{1} \frac{\langle\dot{x}, \dot{x}\rangle}{v^{2}} d s
$$

analogously to the problem of Riemannian geodesics between two given points. This is actually possible although there are some technical problems due to the fact that the constraint equation does not describe a smooth manifold, because of the presence of a square root. Indeed we can approximate the constraint by a sequence of smooth manifolds described by the differential equation

$$
v \dot{v}+\langle D U(x), \dot{x}\rangle+R(v) \sqrt{\langle\dot{x}, \dot{x}\rangle+\varepsilon^{2}}=0
$$

and where the functional $A$ achieves its minimum value since it is bounded from below and it satisfies the Palais-Smale compactness condition (as we can see using the same methods employed to derive the differential equation for the brachistochrones). Then sending $\varepsilon$ to 0 we can obtain the existence of a smooth minimizer for $A$ satisfying the conservation law (14), as in the Riemannian geodesic problem.

However, to obtain the existence of a (minimal) brachistochrone we think that the direct use of $T$ and the constraint equation (5) (both of them invariant by reparametrizations) is a little easier: although the constraint is not in general closed with respect to the week convergence it is possible to show that a (reparametrized) minimizing sequence possesses a subsequence weakly convergent to a couple $(x, v)$ satisfying the constraint equation (5).

The action functional could be very useful to find multiple critical points, but actually their meaning is not completely clear. Indeed, although we have an uniqueness results for minimizers whenever $p$ and $q$ are sufficiently close (see Prop. 3.1), it is not clear if we can consider a curve satisfying (15) as a brachistochrone (as we can do in the case without friction). This is due to the fact that equation (15) is not an ordinary differential equation, but an integro-differential equation where the behaviour of $(x, v)$ at the instant $s \in] 0,1[$ is influenced by an integral evaluated in all the interval $[s, 1]$. In particular we can not say in principle that along a solution of $(15)$, if $x\left(s_{1}\right)$ and $x\left(s_{2}\right)$ are sufficiently close (and $s_{1}<s_{2}$ ), then $x_{\mid\left[s_{1}, s_{2}\right]}$ is a minimizer of $T$ (along curves defined in the interval $\left.\left[s_{1}, s_{2}\right]\right)$. Anyway thanks to the Palais-Smale condition for the action functional, it is possible to obtain multiplicity results for its critical points.

For the brachistochrone problem in a relativistic stationary spacetime (corresponding to the classical autonomous case without friction) the reader is referred to $[2,3,6]$, where the employed techniques allow to deal also with the classical autonomous case.

\section{EXISTENCE OF BRACHISTOCHRONES}

Let us consider a complete Riemannian manifold $\mathcal{M}$ of dimension $n$. Our functional framework will be given by the product of Sobolev spaces

$$
H^{1, p}([0,1], \mathcal{M}) \times H^{1, p}\left([0,1], \mathbb{R}^{+}\right), \quad 1 \leq p \leq \infty .
$$


Here we recall that $H^{1, p}\left([0,1], \mathbb{R}^{n}\right)$ consists of all the absolutely continuous curves $\gamma(s):[0,1] \rightarrow \mathbb{R}^{n}$ such that $\dot{\gamma}(s)$ is in $L^{p}\left([0,1], \mathbb{R}^{n}\right) . H^{1, p}([0,1], \mathcal{M})$ can instead be defined in terms of local charts $(U, \varphi)$ of $\mathcal{M}, U \subset M$, $\varphi: U \rightarrow \mathbb{R}^{n}$,

$$
\begin{aligned}
& H^{1, p}([0,1], \mathcal{M})=\{z:[0,1] \rightarrow \mathcal{M}: \forall(U, \varphi) \text { local chart of } \mathcal{M} \\
& \left.\forall I=\left[t_{1}, t_{2}\right] \subseteq[0,1]: I \subseteq z^{-1}(U) \Rightarrow \varphi \circ z \in H^{1, p}\left(I, \mathbb{R}^{n}\right)\right\}
\end{aligned}
$$

Anyway, for our purposes, we will actually suppose, by Nash embedding theorem [4], that $\mathcal{M}$ is embedded in the Euclidean space $\mathbb{R}^{N}$, for some $N$, in such a way that $\mathcal{M}$ inherits the Riemannian structure of $\mathbb{R}^{N}$. In this way the spaces

$$
L^{p}([0,1], \mathcal{M})
$$

used throughout the paper, are well defined.

Let $p, q$ be two fixed point of the Riemannian manifold $\mathcal{M}$ (we suppose $p \neq q$ ); in order to find the minimal brachistochrone, defined in $[0,1]$, from $p$ to $q$, we recall that the constraint equation satisfied by $x(s)$ and $v(s)$ is

$$
\Phi(x, v)=v \dot{v}+\langle D U(x), \dot{x}\rangle+R(v) \sqrt{\langle\dot{x}, \dot{x}\rangle}=0 \text { a.e. }
$$

where $D U$ is the gradient of the potential energy $U: \mathcal{M} \rightarrow \mathbb{R}$, and $R: \mathbb{R}^{+} \rightarrow \mathbb{R}^{+}$is a non negative function depending on $v$ that represents the frictional force.

Here we assume that both $U$ and $R$ are smooth, and moreover

$$
R(0)=0, \quad \quad \quad \limsup _{v \rightarrow \infty} \frac{R(v)}{v}<+\infty,
$$

and

$$
\limsup _{d_{\mathcal{M}}(x, p) \rightarrow \infty} \frac{-U(x)}{d_{\mathcal{M}}(x, p)}<\infty,
$$

where $d_{\mathcal{M}}(\cdot, \cdot)$ is the Riemannian distance in $\mathcal{M}$.

Let us choose a constant $\Delta>0$ and an initial velocity $v_{0} \geq \Delta$. The space of curves in which we first search the minimum is

$$
\begin{aligned}
\Lambda= & \left\{(x, v) \in H^{1,1}([0,1], \mathcal{M}) \times H^{1,1}\left([0,1], \mathbb{R}^{+}\right): x(0)=p, x(1)=q,\right. \\
& \left.v(0)=v_{0}, v(s) \geq \Delta, \Phi(x, v)=0 \text { a.e. }\right\}
\end{aligned}
$$

The main theorem we will prove in this section is the following:

Theorem 2.1. Under the assumptions (10) and (11), and fixed $\Delta>0$, there exists a positive constant $v_{*}(p, q, \Delta)$ such that for every $v_{0} \geq v_{*}$ there is a minimal brachistochrone, defined in $[0,1]$, from $p$ to $q$ with initial velocity $v_{0}$ and $v(s)>\Delta, \forall s \in[0,1]$. This means that there exists a couple of smooth curves $(x, v) \in \Lambda$ such that

$$
T(x, v)=\int_{0}^{1} \frac{\sqrt{\langle\dot{x}(\tau), \dot{x}(\tau)\rangle}}{v(\tau)} d \tau=\inf _{\Lambda} T
$$

parametrized in such a way that

$$
\frac{\sqrt{\langle\dot{x}(s), \dot{x}(s)\rangle}}{v(s)} \equiv C(x, v)=\text { const. } \quad \text { in }[0,1] .
$$


Moreover, the brachistochrone $(x, v)$ is a couple of $\mathcal{C}^{2}$ curves that satisfies equation (5) and the following equation:

$$
D_{s}\left[\left(\frac{1}{(v(s))^{2}}+\frac{P(s) R(v(s))}{v(s) \sqrt{\langle\dot{x}(s), \dot{x}(s)\rangle}}\right) \dot{x}(s)\right]+\frac{d}{d s}\left(\frac{P(s)}{v(s)}\right) D U(x(s))=0,
$$

where $D_{s}$ is the covariant derivative along the direction $\dot{x}(s)$, and $P(s)$ is the following scalar function depending on $x(s)$ and $v(s)$ :

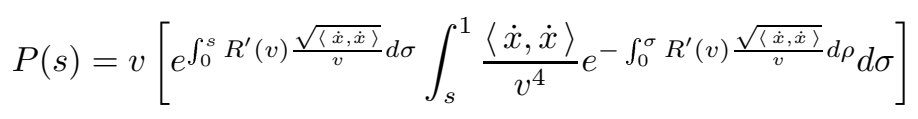

where $R^{\prime}$ denotes the derivative of $R$ with respect to $v$.

Remark 2.2. It can be easily seen that, if (9) and (15) hold for some $\mathcal{C}^{2}$ curves $(x, v)$, then $(x, v)$ is a critical point of $T$ parametrized in such a way that (14) holds. Indeed, expanding expression (15) we have

$$
\left[-\frac{2 \dot{v}}{v^{3}}+\frac{d}{d s}\left(\frac{P}{v}\right) \frac{R}{\sqrt{\langle\dot{x}, \dot{x}\rangle}}+\frac{P R^{\prime}(v) \dot{v}}{v \sqrt{\langle\dot{x}, \dot{x}\rangle}}-\frac{P R(v)}{v} \frac{\left\langle D_{s} \dot{x}, \dot{x}\right\rangle}{\langle\dot{x}, \dot{x}\rangle^{\frac{3}{2}}}\right] \dot{x}+\left[\frac{1}{v^{2}}+\frac{P R(v)}{v \sqrt{\langle\dot{x}, \dot{x}\rangle}}\right] D_{s} \dot{x}+\frac{d}{d s}\left(\frac{P}{v}\right) D U=0 .
$$

Making the scalar product of the left-hand side term of the above expression with $\dot{x}$ and using the constraint equation (5) we get

$$
-\frac{2 \dot{v}}{v^{3}}\langle\dot{x}, \dot{x}\rangle-\frac{d}{d s}\left(\frac{P}{v}\right) v \dot{v}+\frac{P R^{\prime}(v) \dot{v}}{v}\langle\dot{x}, \dot{x}\rangle+\frac{1}{v^{2}}\left\langle D_{s} \dot{x}, \dot{x}\right\rangle=0
$$

But from (16) we have

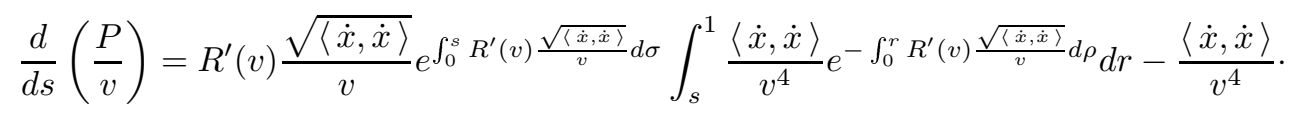

Substituting this expression in (17), with a little algebra we obtain

$$
\frac{1}{v^{2}}\left\langle D_{s} \dot{x}, \dot{x}\right\rangle-\frac{2 \dot{v}}{v^{3}}\langle\dot{x}, \dot{x}\rangle=0
$$

that is

$$
\frac{\langle\dot{x}(s), \dot{x}(s)\rangle}{[v(s)]^{2}} \equiv \text { const. }
$$

Remark 2.3. Let $(x, v)$ a critical point for $T$, and $(a, b) \subset(0,1)$. Let $p_{1}=x(a), q_{1}=x(b), v_{1}=v(a)$, and consider the functional

$$
T_{a, b}(y, w)=\int_{a}^{b} \frac{\sqrt{\langle\dot{y}, \dot{y}\rangle}}{w} d \tau
$$

among all the curves $(y, w)$ such that $y(a)=p_{1}, y(b)=p_{2}, w(a)=v_{1}$, and

$$
\frac{1}{2} w(s)^{2}+U(y(s))+\int_{a}^{s} R(w(\sigma)) \sqrt{\langle\dot{y}(\sigma), \dot{y}(\sigma)\rangle} d \sigma=\frac{1}{2} v_{1}^{2}+U\left(p_{1}\right) .
$$


Then the couple $\left(x_{a, b}=x_{\mid[a, b]}, v_{a, b}=v_{\mid[a, b]}\right)$ satisfies

$$
D_{s}\left[\left(\frac{1}{\left(v_{a, b}(s)\right)^{2}}+\lambda_{a, b}(s) \frac{R\left(v_{a, b}(s)\right)}{\sqrt{\left\langle\dot{x}_{a, b}(s), \dot{x}_{a, b}(s)\right\rangle}}\right) \dot{x}_{a, b}(s)\right]+\frac{d \lambda_{a, b}(s)}{d s} D U\left(x_{a, b}(s)\right)=0,
$$

where

$$
\lambda_{a, b}(s)=\left[e^{\int_{a}^{s} R^{\prime}\left(v_{a, b}\right) \frac{\sqrt{\left\langle\dot{x}_{a, b}, \dot{x}_{a, b}\right\rangle}}{v_{a, b}}} d \sigma \int_{s}^{b} \frac{\left\langle\dot{x}_{a, b}, \dot{x}_{a, b}\right\rangle}{v_{a, b}^{4}} e^{-\int_{a}^{\sigma} R^{\prime}\left(v_{a, b}\right) \frac{\sqrt{\left\langle\dot{x}_{a, b}, \dot{x}_{a, b}\right\rangle}}{v_{a, b}}} d \rho\right],
$$

that is the equation for critical points of $T_{a, b}$. Note that $\lambda_{0,1} \cdot v$ yields $P(s)$ of expression (16).

Here we stress the fact that the parameter used does not represent time and then the choice of the interval $[0,1]$ in the formulation (3) is purely arbitrary, although it obviously has to be consistent with Definition 1.1.

For our aim, we will prove several lemmas in this section; we will first show that the class $\Lambda$ is not empty (Lem. 2.4), and then we will use a minimizing sequence in $\Lambda$ that we will show (Lem. 2.6) to be uniformly bounded in the space

$$
H^{1, \infty}([0,1], \mathcal{M}) \times H^{1, \infty}\left([0,1], \mathbb{R}^{+}\right) .
$$

We will them show the existence of a minimizer of $T$ in the set $\Lambda$ belonging to the space (23), that can be reparametrized in such a way that (14) holds (Lem. 2.7).

Then, in order to write Lagrange's equation, we will also show that the velocity curve $v(s)$ is strictly bounded away from $\Delta$ for an opportune choice of the initial value $v_{0}$ (Lem. 2.9). At the end of the section the regularity of the solution (Lem. 2.11) and Lagrange's equation (15) will be proven.

We now start the proof by showing the following:

Lemma 2.4. There exists a constant $v_{*}$ such that $\forall v_{0} \geq v_{*}$ the class $\Lambda$ is not empty.

Proof. Let $z(s)$ be the curve in $\mathcal{M}$ that minimizes the Riemannian distance from $p$ to $q$; for each choice of $v_{0}$ there exists a solution $w(s)$ of the Cauchy problem

$$
\left\{\begin{array}{l}
\Phi(z, w) \equiv w \dot{w}+\langle D U(z), \dot{z}\rangle+R(w) \sqrt{\langle\dot{z}, \dot{z}\rangle}=0, \\
w(0)=v_{0} .
\end{array}\right.
$$

From the constraint equation, written as in (6),

$$
\frac{1}{2} w^{2}(s)+U(z(s))+\int_{0}^{s} R(w(\tau)) \sqrt{\langle\dot{z}(\tau), \dot{z}(\tau)\rangle} d \tau=E,
$$

where

$$
E=\frac{1}{2} v_{0}^{2}+U(p)
$$

we have, for some constant $a_{1}$,

$$
\frac{1}{2} w^{2} \leq E-U(z) \leq a_{1}+\frac{1}{2} v_{0}^{2},
$$

and then

$$
w \leq v_{0}+a_{2}
$$


for some constant $a_{2}$. Using $(10,25,26)$ and (28) we also have, for some constants $a_{3}, a_{4}$,

$$
U(p)+\frac{1}{2} v_{0}^{2} \leq \frac{1}{2} w^{2}+\left(a_{3} v_{0}+a_{4}\right)+U(z),
$$

thus

$$
\frac{1}{2} w^{2} \geq \frac{1}{2} v_{0}^{2}-a_{3} v_{0}+a_{5}
$$

where $a_{5}$ is another constant. Note that all the constants here considered are independent from $v_{0}$. From the above relation we can conclude that

$$
\inf _{[0,1]} w \underset{v_{0} \rightarrow \infty}{\longrightarrow} \infty
$$

and then, fixed $\Delta>0, \Lambda \neq \emptyset$ for some $v_{*}$ opportunely chosen.

Remark 2.5. By the way, it is easily seen from

$$
\inf _{\Lambda} T \leq \int_{0}^{1} \frac{\sqrt{\langle\dot{z}, \dot{z}\rangle}}{w} d \tau \leq \frac{1}{\inf _{[0,1]} w} \int_{0}^{1} \sqrt{\langle\dot{z}, \dot{z}\rangle} d \tau
$$

that

$$
\inf _{\Lambda} T \leq \frac{b_{1}}{v_{0}+b_{2}}
$$

for some constants $b_{1}, b_{2}$ depending on $p, q$ and $U$. In particular

$$
\inf _{\Lambda} T \underset{v_{0} \rightarrow \infty}{\longrightarrow} 0
$$

These estimates will be useful later in Lemma 2.9.

We now fix $v_{0}$ as in the previous lemma, and prove the existence of a minimizing sequence belonging to the space $(23)$.

Lemma 2.6. There exists a minimizing sequence of $T$ consisting of curves $\left(x_{n}, v_{n}\right)$ uniformly bounded in

$$
H^{1, \infty}([0,1], \mathcal{M}) \times H^{1, \infty}\left([0,1], \mathbb{R}^{+}\right)
$$

and satisfying the constraint equation (9), that is

$$
\begin{gathered}
\lim _{n \rightarrow \infty} \int_{0}^{1} \frac{\sqrt{\left\langle\dot{x}_{n}, \dot{x}_{n}\right\rangle}}{v_{n}}=\inf _{\Lambda} T(x, v), \\
v_{n} \dot{v}_{n}+\left\langle D U\left(x_{n}\right), \dot{x}_{n}\right\rangle+R\left(v_{n}\right) \sqrt{\left\langle\dot{x}_{n}, \dot{x}_{n}\right\rangle}=0 \quad \text { a.e. }
\end{gathered}
$$

Proof. We can suppose the minimizing sequence to be of class $\mathcal{C}^{1}$. There obviously exists a constant $K$ such that

$$
T\left(x_{n}, v_{n}\right) \leq K
$$


As in (25), we can write

$$
\frac{1}{2} v_{n}^{2}+U\left(x_{n}\right)+\int_{0}^{s} R\left(v_{n}\right) \sqrt{\left\langle\dot{x}_{n}, \dot{x}_{n}\right\rangle} d \tau=E,
$$

and thanks to the assumption (11) made on $U$ we have some constants $a, b$ such that

$$
\frac{1}{2} v_{n}^{2} \leq E-U\left(x_{n}\right) \leq E+a+b d_{\mathcal{M}}(x, p)
$$

holds. Therefore

$$
\begin{aligned}
\frac{1}{2}\left\|v_{n}\right\|_{\infty}^{2} & \leq E+a+b \int_{0}^{1} \sqrt{\left\langle\dot{x}_{n}, \dot{x}_{n}\right\rangle} d \tau \leq E+a+b\left\|v_{n}\right\|_{\infty} \int_{0}^{1} \frac{\sqrt{\left\langle\dot{x}_{n}, \dot{x}_{n}\right\rangle}}{v_{n}} d \tau \\
& \leq E+a+b K\left\|v_{n}\right\|_{\infty}
\end{aligned}
$$

from which we deduce the existence of a constant $d>0$ such that

$$
v_{n} \leq d, \quad \forall n
$$

Thus

$$
\int_{0}^{1} \sqrt{\left\langle\dot{x}_{n}, \dot{x}_{n}\right\rangle} d \tau \leq \beta \quad \forall n
$$

for an opportune constant $\beta>0$.

Let us now define a sequence of curves $y_{n}(s)=x_{n}\left(\lambda_{n}(s)\right)$, where $\lambda_{n}(s)$ solves the Cauchy problem

$$
\left\{\begin{array}{l}
\dot{\lambda}_{n}(s)=\frac{\int_{0}^{1}\left(\sqrt{\left\langle\dot{x}_{n}, \dot{x}_{n}\right\rangle}+1\right) d \tau}{\sqrt{\left\langle\dot{x}_{n}\left(\lambda_{n}(s)\right), \dot{x}_{n}\left(\lambda_{n}(s)\right)\right\rangle}+1} \\
\lambda_{n}(0)=0
\end{array}\right.
$$

It can be easily seen, with a continuity argument, that there exists $s_{n} \in \mathbb{R}^{+}$such that $\lambda_{n}\left(s_{n}\right)=1$; indeed, $\lambda_{n}$ is increasing and if there was not such a $s_{n}$ it would be

$$
\lim _{s \rightarrow \infty} \dot{\lambda}_{n}(s)=0
$$

and this is absurd due to the upper limitation for the denominator in the right-hand side term of the differential equation, that holds because $\dot{x}_{n}$ is $\mathcal{C}^{0}$.

From (38) we have

$$
\int_{0}^{s_{n}} \dot{\lambda}_{n}\left(\sqrt{\left\langle\dot{x}_{n}\left(\lambda_{n}\right), \dot{x}_{n}\left(\lambda_{n}\right)\right\rangle}+1\right) d s=\left[\int_{0}^{1}\left(\sqrt{\left\langle\dot{x}_{n}, \dot{x}_{n}\right\rangle}+1\right) d s\right] s_{n}
$$

then, introducing the variable change

$$
\lambda_{n}(s)=\sigma
$$

expression (39) becomes

$$
\int_{0}^{1}\left(\sqrt{\left\langle\dot{x}_{n}, \dot{x}_{n}\right\rangle}+1\right) d \sigma=\left[\int_{0}^{1}\left(\sqrt{\left\langle\dot{x}_{n}, \dot{x}_{n}\right\rangle}+1\right) d \sigma\right] s_{n}
$$


giving

$$
s_{n}=1 .
$$

Then $\lambda_{n}(s)$ is a reparametrization of $[0,1]$, and from (37) the curves $y_{n}$ are uniformly bounded; differentiating $y_{n}$ and using (37) again we obtain

$$
\dot{y}_{n}=\dot{x}_{n}\left(\lambda_{n}\right) \dot{\lambda}_{n} \leq(\beta+1) \frac{\dot{x}_{n}\left(\lambda_{n}\right)}{\sqrt{\left\langle\dot{x}_{n}, \dot{x}_{n}\right\rangle}+1},
$$

thus the curves $y_{n}$ are uniformly bounded in the $H^{1, \infty}$-norm. Now, setting

$$
w_{n}(s)=v_{n}\left(\lambda_{n}(s)\right),
$$

we have

$$
\dot{w}_{n} w_{n}+\left\langle D U\left(y_{n}\right), \dot{y}_{n}\right\rangle+R\left(w_{n}\right) \sqrt{\left\langle\dot{y}_{n}, \dot{y}_{n}\right\rangle}=\left[\dot{v}_{n} v_{n}+\left\langle D U\left(x_{n}\right), \dot{x}_{n}\right\rangle+R\left(v_{n}\right) \sqrt{\left\langle\dot{x}_{n}, \dot{x}_{n}\right\rangle}\right] \dot{\lambda}_{n},
$$

and so the couple $\left(y_{n}, w_{n}\right)$ satisfies the constraint equation (9) and then it belongs to $\Lambda$. From the expression of the constraint itself it can also be seen that $w_{n}$ are uniformly bounded in the $H^{1, \infty}$-norm, and in conclusion, since $T$ is invariant by reparametrizations, the sequence $\left(y_{n}, w_{n}\right)$ is a minimizing sequence in $H^{1, \infty}$.

We are now ready to show the existence of a minimal brachistochrone.

Lemma 2.7. There exists a minimizer $(x, v)$ of $T$ in the set $\Lambda$, reparametrized in such a way that (14) holds almost everywhere.

Proof. By the above lemmas we can suppose there exists a sequence of curves $\left(x_{n}, v_{n}\right)$, uniformly bounded in $H^{1, \infty}$, and uniformly converging to a couple $(x, v)$. We first have to show that $(x, v)$ is the required minimizer.

Since the curves $v_{n}$ are uniformly bounded away from zero we have

$$
\int_{0}^{1} \frac{\sqrt{\left\langle\dot{x}_{n}, \dot{x}_{n}\right\rangle}}{v_{n}} d \tau-\int_{0}^{1} \frac{\sqrt{\left\langle\dot{x}_{n}, \dot{x}_{n}\right\rangle}}{v} d \tau \underset{n \rightarrow \infty}{\longrightarrow} 0
$$

but the $L^{\infty}$ uniform boundness of $\dot{x}_{n}$ and $\dot{v}_{n}$ implies (we recall that we work in a local chart, by Nash theorem [4])

$$
\begin{aligned}
& \dot{x}_{n} \rightarrow \dot{x} \quad \text { in } L^{1}\left([0,1], \mathbb{R}^{N}\right), \\
& \dot{v}_{n} \rightarrow \dot{v} \quad \text { in } L^{1}([0,1], \mathbb{R}),
\end{aligned}
$$

and then

$$
\int_{0}^{1} \frac{\sqrt{\langle\dot{x}, \dot{x}\rangle}}{v} d \tau \leq \lim _{n \rightarrow \infty} \inf \int_{0}^{1} \frac{\sqrt{\left\langle\dot{x}_{n}, \dot{x}_{n}\right\rangle}}{v} d \tau
$$

(cf. [1]). Combining (43) and (45) we obtain

$$
T(x, v) \leq \inf _{\Lambda} T,
$$

and so we still have to show that the couple $(x, v)$ satisfies almost everywhere the constraint equation (9) to prove that the equality holds in (46). 
For every closed interval $\left[t_{1}, t_{2}\right] \subset[0,1]$ we have

$$
0=\int_{t_{1}}^{t_{2}} \dot{v}_{n} d \tau+\int_{t_{1}}^{t_{2}} \frac{R\left(v_{n}\right)}{v_{n}} \sqrt{\left\langle\dot{x}_{n}, \dot{x}_{n}\right\rangle} d \tau+\int_{t_{1}}^{t_{2}}\left\langle\frac{D U\left(x_{n}\right)}{v_{n}}, \dot{x}_{n}\right\rangle d \tau,
$$

and, using (44), it follows that

$$
\int_{0}^{1} \chi_{\left[t_{1}, t_{2}\right]}\left(\dot{v}+\frac{R(v)}{v} \sqrt{\langle\dot{x}, \dot{x}\rangle}+\left\langle\frac{D U(x)}{v}, \dot{x}\right\rangle\right) d \tau \leq 0,
$$

where $\chi_{\left[t_{1}, t_{2}\right]}$ is the characteristic function of the interval $\left[t_{1}, t_{2}\right]$, and then

$$
\dot{v}(s)+\frac{R(v(s))}{v} \sqrt{\langle\dot{x}(s), \dot{x}(s)\rangle}+\left\langle\frac{D U(x(s))}{v(s)}, \dot{x}(s)\right\rangle \leq 0, \quad \text { a.e. in }[0,1] .
$$

We define now the curve $w$ solution of the Cauchy problem

$$
\left\{\begin{array}{l}
\dot{w}=-\frac{R(w)}{w} \sqrt{\langle\dot{x}, \dot{x}\rangle}-\left\langle\frac{D U(x)}{w}, \dot{x}\right\rangle \\
w(0)=v_{0}
\end{array}\right.
$$

The function $w$ is defined in the interval $[0,1]$ and is such that

$$
w(0)=v(0)
$$

then, by a simple comparison argument in ODE, it is

$$
w(t) \geq v(t) \quad \forall t \in[0,1] .
$$

But if the strict equality held in (51) it would be

$$
T(x, w)<\int_{0}^{1} \frac{\sqrt{\langle\dot{x}, \dot{x}\rangle}}{v} d \tau \leq \inf _{\Lambda} T,
$$

and since $(x, w) \in \Lambda$ (recall that $w(s) \geq \Delta, \forall s \in[0,1]$ ) we would obtain a contradiction. Then

$$
v=w
$$

and $(x, v) \in \Lambda$. We have showed the first part of the lemma.

Let us now define, $\forall \varepsilon>0$ the curve

$$
x_{\varepsilon}(s)=x\left(\lambda_{\varepsilon}(s)\right),
$$

where $\lambda_{\varepsilon}$ solves the Cauchy problem

$$
\left\{\begin{array}{l}
\dot{\lambda}_{\varepsilon}(s)=\frac{\int_{0}^{1} \frac{\sqrt{\langle\dot{x}, \dot{x}\rangle}}{v} d \tau+\varepsilon}{\frac{\sqrt{\left\langle\dot{x}\left(\lambda_{\varepsilon}(s), \dot{x}\left(\lambda_{\varepsilon}(s)\right)\right\rangle\right.}}{v\left(\lambda_{\varepsilon}(s)\right)}+\varepsilon} \\
\lambda_{\varepsilon}(0)=0 .
\end{array}\right.
$$


Since $\frac{\sqrt{\left\langle\dot{x}\left(\lambda_{\varepsilon}(s) \dot{x}\left(\lambda_{\varepsilon}(s)\right)\right\rangle\right.}}{v\left(\lambda_{\varepsilon}(s)\right)}+\varepsilon$ is uniformly bounded, whenever $s \in[0,1]$, with a similar argument as in Lemma 2.6 it can be shown that

$$
\lambda_{\varepsilon}(1)=1
$$

moreover, differentiating both terms in (52) and using (53) it can be easily seen that the curves $x_{\varepsilon}$ are uniformly bounded in the $H^{1, \infty}$ norm, then there exists a curve $z$ such that

$$
\begin{array}{ll}
x_{\varepsilon} \underset{\varepsilon \rightarrow 0}{\longrightarrow} z & \text { in } L^{1}\left([0,1], \mathbb{R}^{N}\right), \\
\dot{x}_{\varepsilon} \underset{\varepsilon \rightarrow 0}{\longrightarrow} \dot{z} & \text { in } L^{1}\left([0,1], \mathbb{R}^{N}\right) .
\end{array}
$$

If we now define $v_{\varepsilon}$ to be the solution of the Cauchy problem

$$
\left\{\begin{array}{l}
\dot{v}_{\varepsilon}=-\frac{R\left(v_{\varepsilon}\right)}{v_{\varepsilon}} \sqrt{\left\langle\dot{x}_{\varepsilon}, \dot{x}_{\varepsilon}\right\rangle}-\left\langle\frac{D U(x)}{v_{\varepsilon}}, \dot{x}_{\varepsilon}\right\rangle, \\
v_{\varepsilon}(0)=v_{0}
\end{array}\right.
$$

we find

$$
v_{\varepsilon}(s)=v\left(\lambda_{\varepsilon}(s)\right)
$$

because they both solve the same Cauchy problem. Then the curves $v_{\varepsilon}$ are uniformly bounded in $H^{1, \infty}$, and there exists $w$ such that

$$
v_{\varepsilon} \underset{\varepsilon \rightarrow 0}{\longrightarrow} w \text { uniformly }
$$

Now

$$
\frac{\sqrt{\left\langle\dot{x}_{\varepsilon}, \dot{x}_{\varepsilon}\right\rangle}}{v_{\varepsilon}}=\left(\int_{0}^{1} \frac{\sqrt{\langle\dot{x}, \dot{x}\rangle}}{v} d \tau+\varepsilon\right) \frac{\frac{\sqrt{\langle\dot{x}, \dot{x}\rangle}}{v}}{\frac{\sqrt{\langle\dot{x}, \dot{x}\rangle}}{v}+\varepsilon},
$$

therefore, $\forall s \in[0,1]$,

$$
\limsup _{\varepsilon \rightarrow 0} \frac{\sqrt{\left\langle\dot{x}_{\varepsilon}, \dot{x}_{\varepsilon}\right\rangle}}{v_{\varepsilon}} \leq \int_{0}^{1} \frac{\sqrt{\langle\dot{x}, \dot{x}\rangle}}{v} d \tau=\inf _{\Lambda} T,
$$

and then, by (54) and (56) we have, for every closed interval $\left[t_{1}, t_{2}\right] \subset[0,1]$,

$$
\int_{t_{1}}^{t_{2}} \frac{\sqrt{\langle\dot{z}, \dot{z}\rangle}}{w} d \tau \leq\left(t_{2}-t_{1}\right) \inf _{\Lambda} T
$$

Arguing as in the first part of this lemma we obtain

$$
\frac{\sqrt{\langle\dot{z}, \dot{z}\rangle}}{w} \leq \inf _{\Lambda} T \quad \text { a.e. in }[0,1]
$$

and as done in (51) we find that $w$ is a subsolution of the Cauchy problem

$$
\left\{\begin{array}{l}
\dot{u}=-\frac{R(u)}{u} \sqrt{\langle\dot{z}, \dot{z}\rangle}-\left\langle\frac{D U(z)}{u}, \dot{z}\right\rangle \\
u(0)=v_{0}
\end{array}\right.
$$


then, if $u$ is the solution of the above problem, we have

$$
u \geq w \quad \forall s \in[0,1]
$$

and from (59) it immediately follows by integration in $[0,1]$ that $u=v$ and

$$
\frac{\sqrt{\langle\dot{z}, \dot{z}\rangle}}{w}=\inf _{\Lambda} T \quad \text { a.e. in }[0,1]
$$

In conclusion $(z, w) \in \Lambda$ is such that (62) holds and

$$
T(z, w)=\min _{\Lambda} T
$$

Remark 2.8. Note that in particular we have

$$
\sqrt{\langle\dot{z}, \dot{z}\rangle}=v \inf _{\Lambda} T \geq \Delta \cdot \inf _{\Lambda} T>0 .
$$

Let us now denote by $(x, v)$ a minimizer of $T$ in $\Lambda$ reparametrized in such a way that (14) holds. In order to write Lagrange's equation, we first show that the velocity curve $v(s)$ is bounded away from $\Delta$ for an opportune choice of the initial velocity $v_{0}$. This is achieved by the following:

Lemma 2.9. There exists a constant $v_{*}$, depending on $\Delta$ such that, for every $v_{0} \geq v_{*}$, the couple $(x, v)$ is such that

$$
v(s)>\Delta, \quad \forall s \in[0,1] .
$$

Proof. The constraint equation already used in (25) and (34), yields

$$
\frac{1}{2} v^{2}+U(x) \leq \frac{1}{2} v_{0}^{2}+U(p),
$$

because $R(v)$ is a non negative function. Using (11) we find, for some constants $a$ and $b$,

$$
\frac{1}{2} v^{2} \leq \frac{1}{2} v_{0}^{2}+U(p)+a+b\|v\|_{\infty} \int_{0}^{1} \frac{\sqrt{\langle\dot{x}, \dot{x}\rangle}}{v} d \tau
$$

and then, from (14),

$$
\frac{1}{2}\|v\|_{\infty}^{2} \leq \frac{1}{2} v_{0}^{2}+b\|v\|_{\infty} T(x, v)+U(p)+a,
$$

and so there is a constant $h$ such that

$$
\|v\|_{\infty} \leq v_{0}+h
$$

We now observe, from (30) and (67), that

$$
\frac{1}{v_{0}+h} \int_{0}^{1} \sqrt{\langle\dot{x}, \dot{x}\rangle} d s \leq \int_{0}^{1} \frac{\sqrt{\langle\dot{x}, \dot{x}\rangle}}{v} d s=\inf _{\Lambda} T \leq \frac{b_{1}}{v_{0}+b_{2}}
$$


and then there exists a compact set $\mathcal{K} \subset \mathcal{M}$, not depending on the initial velocity $v_{0}$, such that the curve $x(s) \in \mathcal{K}$. This means that $|U(x)|<h_{U}$ for some constant $h_{U}$ independent from $v_{0}$. Using again the constraint equation together with (10) and (11) it follows

$$
\begin{aligned}
\frac{1}{2} v_{0}^{2}+U(p)= & \frac{1}{2} v^{2}+\int_{0}^{s} R(v) \sqrt{\langle\dot{x}, \dot{x}\rangle} d \tau+U(x) \leq \frac{1}{2} v^{2}+\int_{0}^{1} v R(v) \frac{\sqrt{\langle\dot{x}, \dot{x}\rangle}}{v} d \tau+h_{U} \\
& \leq \frac{1}{2} v^{2}+h_{1}\|v\|_{\infty}^{2} T(x, v)+h_{U},
\end{aligned}
$$

for some constant $h_{1}$ depending on $R(v)$. Taking into account (67) we obtain

$$
\frac{1}{2} v^{2} \geq\left[\frac{1}{2}-h_{1} T(x, v)\right] v_{0}^{2}+\left(k_{1} v_{0}+k_{2}\right) T(x, v)+k_{3}
$$

for some constants $k_{i}, i=1,2,3$. Then, recalling (31) of Remark 2.5, we have

$$
\inf _{[0,1]} v \underset{v_{0} \rightarrow \infty}{\longrightarrow} \infty
$$

and there exists a constant $v_{*}$, depending on $p, q, \Delta$ and, of course, on the data $R$ and $U$ of the problem, that ensures that lemma holds.

Remark 2.10. We observe that it has so far been shown the existence of the minimum in $\Lambda$. Actually, the brachistochrone with initial velocity $v_{0}$ could not belong to the space $\Lambda$, that is there could be $(\tilde{x}, \tilde{v})$ such that

$$
T(\tilde{x}, \tilde{v})<\inf _{\Lambda} T, \quad \inf _{[0,1]} \tilde{v} \leq \Delta .
$$

But the argument of the previous lemma can be repeated, using the first relation above, obtaining a contradiction with the second one. Then the minimizer in $\Lambda$ is actually the sought brachistochrone.

Let us now complete the proof of the main theorem.

Proof of Theorem 2.1. By Lemma 2.9 if $(x, v)$ is a minimizer we have

$$
\int_{0}^{1} \delta\left(\frac{\sqrt{\langle\dot{x}, \dot{x}\rangle}}{v}\right)[\zeta, \omega]=0
$$

for every couple $(\zeta, \omega)$ of $\mathcal{C}^{1}$ admissible variations such that $\zeta(0)=\zeta(1)=0$ and $\omega(0)=\omega(1)=0$. More precisely, thanks to the Nash embedding theorem we can assume that $\mathcal{M}$ is embedded in some $\mathbb{R}^{N}$ and $\langle\cdot, \cdot\rangle$ is the Euclidean structure of $\mathbb{R}^{N}$. Therefore, since $v(s)>\Delta$ for any $s \in[0,1]$ and $(x, v)$ is a minimizer we have:

$$
\begin{aligned}
0 & =\lim _{\varepsilon \rightarrow 0} \int_{0}^{1} \frac{1}{\varepsilon}\left(\frac{\sqrt{\langle\dot{x}+\varepsilon \dot{\zeta}, \dot{x}+\varepsilon \dot{\zeta}\rangle}}{v+\varepsilon \omega}-\frac{\sqrt{\langle\dot{x}, \dot{x}\rangle}}{v}\right) d \tau \\
& =\lim _{\varepsilon \rightarrow 0} \int_{0}^{1} \frac{(\sqrt{\langle\dot{x}+\varepsilon \dot{\zeta}, \dot{x}+\varepsilon \dot{\zeta}\rangle}-\sqrt{\langle\dot{x}, \dot{x}\rangle})}{\varepsilon(v+\varepsilon \omega)}-\frac{\sqrt{\langle\dot{x}, \dot{x}\rangle}}{v(v+\varepsilon \omega)} \omega d \tau \\
& =\lim _{\varepsilon \rightarrow 0} \int_{0}^{1} \frac{2\langle\dot{x}, \dot{\zeta}\rangle+\varepsilon\langle\dot{\zeta}, \dot{\zeta}\rangle}{(v+\varepsilon \omega)(\sqrt{\langle\dot{x}+\varepsilon \dot{\zeta}, \dot{x}+\varepsilon \dot{\zeta}\rangle}+\sqrt{\langle\dot{x}, \dot{x}\rangle})}-\frac{\sqrt{\langle\dot{x}, \dot{x}\rangle}}{v(v+\varepsilon \omega)} \omega d \tau,
\end{aligned}
$$


where we use $v>\Delta$, (14) (satisfied a.e.) and the fact that the variations are $\mathcal{C}^{1}$. Recalling Lebesgue theorem on dominate convergence for $\varepsilon \rightarrow 0$, the above expression becomes

$$
\int_{0}^{1} \frac{\langle\dot{x}, \dot{\zeta}\rangle}{v \sqrt{\langle\dot{x}, \dot{x}\rangle}}-\frac{\sqrt{\langle\dot{x}, \dot{x}\rangle}}{v^{2}} \omega d \tau=0
$$

Observing that $\dot{x} \in T_{x} \mathcal{M}$ and introducing the covariant derivative $D_{s}$ in $\mathcal{M}$ along the direction $\dot{x}(s)$, in the above expression we can use the projection $D_{s} \zeta$ of $\dot{\zeta}$ on $T_{x} \mathcal{M}$, from which, using (14) again, it is easily seen that

$$
\int_{0}^{1} \frac{\left\langle\dot{x}, D_{s} \zeta\right\rangle}{v^{2}}-\frac{\langle\dot{x}, \dot{x}\rangle}{v^{3}} \omega d s=0
$$

In order to find the admissible variations, we first observe that the constraint is only given by

$$
\Phi(x, v)=0
$$

because the strict inequality in (65) of last lemma showed that the velocity curve $v(s)$ is bounded away from $\Delta$ for $v_{0}$ greater than some opportune constant $v_{*}$. From $(73)$ we have

$$
0=d \Phi(x, v)[\zeta, \omega]=\omega \dot{v}+v \dot{\omega}+\left\langle H^{U}(x)[\zeta], \dot{x}\right\rangle+\left\langle D U(x), D_{s} \zeta\right\rangle+R^{\prime}(v) \omega \sqrt{\langle\dot{x}, \dot{x}\rangle}+R(v) \frac{\left\langle\dot{x}, D_{s} \zeta\right\rangle}{\sqrt{\langle\dot{x}, \dot{x}\rangle}}
$$

where $H^{U}(x)$ is the Hessian of $U$, that can be defined in terms of any geodesic curve $\gamma(s)$ such that $\gamma(0)=x$ and $\dot{\gamma}(0)=\xi$ :

$$
\left\langle H^{U}(x)[\xi], \xi\right\rangle=\left(\frac{d}{d s}\right)^{2} U(\gamma(s))
$$

and $R^{\prime}(v)$ denotes the derivative of $R$ with respect to the argument $v$. Defining

$$
\begin{aligned}
A(x, v, \zeta) & =\frac{1}{v}\left(\left\langle H^{U}(x)[\zeta], \dot{x}\right\rangle+\left\langle D U(x), D_{s} \zeta\right\rangle+\frac{R(v)\left\langle\dot{x}, D_{s} \zeta\right\rangle}{\sqrt{\langle\dot{x}, \dot{x}\rangle}}\right) \\
B(x, v) & =\frac{\dot{v}+R^{\prime}(v) \sqrt{\langle\dot{x}, \dot{x}\rangle}}{v}
\end{aligned}
$$

we find that $\omega$ is the solution of the Cauchy problem

$$
\left\{\begin{array}{l}
\dot{\omega}(s)+B(x(s), v(s)) \omega(s)=-A(x(s), v(s), \zeta(s)) \\
\omega(0)=0
\end{array}\right.
$$

and then

$$
\omega(s)=-e^{-\int_{0}^{s} B(\sigma) d \sigma} \int_{0}^{s} A(r) e^{\int_{0}^{r} B(\sigma) d \sigma} d r .
$$

Substituting (78) into (72) gives

$$
0=\int_{0}^{1} \frac{\left\langle\dot{x}, D_{s} \zeta\right\rangle}{v^{2}}-\frac{\langle\dot{x}, \dot{x}\rangle}{v^{3}} \omega d s=\int_{0}^{1}\left\langle\frac{\dot{x}}{v^{2}}, D_{s} \zeta\right\rangle+\frac{\langle\dot{x}, \dot{x}\rangle}{v^{3}} e^{-\int_{0}^{s} B d \sigma}\left(\int_{0}^{s} A(r) e^{\int_{0}^{r} B d \sigma} d r\right) d s
$$


If we define $\alpha(s)$ to be the function

$$
\alpha(s)=\frac{\langle\dot{x}, \dot{x}\rangle}{v^{3}} e^{-\int_{0}^{s} B d \sigma},
$$

last addendum in the right-hand side term of (79) simplifies as follows:

$$
\int_{0}^{1} \alpha(s) \int_{0}^{s} A(r) e^{\int_{0}^{r} B(\sigma) d \sigma} d r d s=\int_{0}^{1} A(r) e^{\int_{0}^{r} B(\sigma) d \sigma} \int_{r}^{1} \alpha(\rho) d \rho d r=\int_{0}^{1} P(s) A(s) d s,
$$

where $P(s)$ is the positive and continue function

$$
P(s)=e^{\int_{0}^{s} B(\sigma) d \sigma} \int_{s}^{1} \alpha(\sigma) d \sigma .
$$

It is easily seen that $P(s)$ has the form (16). The following lemma now shows the regularity of the minimizer $(x, v)$.

Lemma 2.11. The minimizer $(x, v)$ of $T$ in $\Lambda$ is $\mathcal{C}^{2}$.

Proof. We will exploit a bootstrap argument; in view of $(80,81)$ and $(82)$ we have in $(79)$, for $\omega=0$,

$$
\int_{0}^{1}\left\langle\left(\frac{1}{v^{2}}+\frac{P R}{v \sqrt{\langle\dot{x}, \dot{x}\rangle}}\right) \dot{x}-\mathcal{H}(s), D_{s} \zeta\right\rangle d s=0
$$

where

$$
\mathcal{H}(s)=-\frac{P(s)}{v(s)} D U(x(s))+\int_{0}^{s} \frac{P(\sigma)}{v(\sigma)} H^{U}(x(\sigma))[\dot{x}(\sigma)] d \sigma
$$

then, using (14), we obtain

$$
\left(\frac{1}{v^{2}}+\frac{P R}{C v^{2}}\right) \dot{x}=\mathcal{H}+\chi
$$

where $C$ denotes the constant in (14), and $\chi$ is a function such that

$$
D_{s} \chi=0 \text {. }
$$

Then, from the coordinate expression of $D_{s} \chi$ it can be easily seen that $\dot{\chi}$ is $L^{1}$ and then $\chi$ is continue.

Observing that the coefficient of $\dot{x}$ in (85) is bounded away from zero and that, from (14) and (16),

$$
\frac{P(s)}{v(s)}=C^{2} e^{C \int_{0}^{s} R^{\prime}(v(\sigma)) d \sigma} \int_{s}^{1} \frac{1}{[v(\sigma)]^{2}} e^{-C \int_{0}^{\sigma} R^{\prime}(v(\rho)) d \rho} d \sigma
$$

is a $\mathcal{C}^{1}$ function (84) and (85) ensures that $\dot{x}$ is $\mathcal{C}^{0}$ and then $x$ is $\mathcal{C}^{1}$. This implies the following facts: first, $\mathcal{H}$ and $\chi$ are $\mathcal{C}^{1}$ (to prove the second fact we need again the coordinate expression of $D_{s} \chi$ ), and, taking into account the constraint equation written as

$$
\dot{v}=-\frac{1}{v}\langle D U(x), \dot{x}\rangle-C R(v)
$$

where again (14) has been used, $\dot{v}$ is $\mathcal{C}^{0}$ and then $v$ is $\mathcal{C}^{1}$. From (85) again we have that $\dot{x}$ is $\mathcal{C}^{1}$ and then, from the constraint (86), $\dot{v}$ too is $\mathcal{C}^{1}$, obtaining the desired regularity. 
We are now ready to write Lagrange's equation and to conclude the proof of Theorem 2.1. This is easily achieved using (79), that can be written as follows:

$$
\int_{0}^{1}\left\langle\frac{\dot{x}}{v^{2}}, D_{s} \zeta\right\rangle+\frac{P}{v}\left\langle H^{U}(x)[\dot{x}], \zeta\right\rangle+\frac{P}{v}\left\langle\frac{R(v) \dot{x}}{\sqrt{\langle\dot{x}, \dot{x}\rangle}}+D U(x), D_{s} \zeta\right\rangle d s=0,
$$

and we straightforwardly obtain equation (15) by a simple integration by parts.

The proof is complete.

\section{UNIQUENESS OF LOCAL MINIMIZERS}

In this section local uniqueness of critical points of the functional (3) satisfying the constraint equation (5) will be studied. Let us fix $p, q$, which will be considered sufficiently close, and let us fix $v_{0}>0$. With the position

$$
z(t)=\dot{x}(t)
$$

equation (15) is written as

$$
D_{s}\left[\left(\frac{1}{v^{2}}+\frac{\lambda R}{\sqrt{\langle z, z\rangle}}\right) z\right]+\dot{\lambda} D U(x)=0,
$$

where

$$
\lambda(s)=\frac{P(s)}{v(s)}=e^{\int_{0}^{s} R^{\prime}(v) \frac{\sqrt{\langle z, z\rangle}}{v} d \sigma} \int_{s}^{1} \frac{\langle z, z\rangle}{v^{4}} e^{-\int_{0}^{\sigma} R^{\prime}(v) \frac{\sqrt{\langle z, z\rangle}}{v}} d \rho d \sigma
$$

and $v=v(x(s), z(s))$ functionally depends on $(x, z)$ by means of the constraint equation rewritten as

$$
\frac{1}{2} v^{2}+\int_{0}^{s} R(v) \sqrt{\langle z, z\rangle} d \tau+U(x)=\frac{1}{2} v_{0}^{2}+U(p) .
$$

Since we want to consider brachistochrone curves joining $p$ with a close point $q$ we can work in a local chart of $p$ and set $p=0$. With such a fixed coordinate system, (89) becomes

$$
\left[\left(\frac{1}{v^{2}}+\frac{\lambda R}{\sqrt{\langle z, z\rangle}}\right) z\right]^{\prime}=\Gamma(x)\left[z,\left(\frac{1}{v^{2}}+\frac{\lambda R}{\sqrt{\langle z, z\rangle}}\right) z\right]-\dot{\lambda} D U(x)=0,
$$

where $\Gamma(x)$ is the bilinear form induced at the point $x$ by the Christoffel symbols of the metric $\langle\cdot, \cdot\rangle$.

Let us now consider the map

$$
F: \mathcal{C}^{0}\left([0,1], \mathbb{R}^{2 n}\right) \times \mathbb{R}^{n} \rightarrow \mathcal{C}^{0}\left([0,1], \mathbb{R}^{2 n}\right)
$$

that sends the point $(z, x, w)$ into the couple of functions $\left(F_{1}(z, x, w), F_{2}(z, x, w)\right)$, where

$$
\begin{aligned}
& F_{1}(z, x, w)=\left(\frac{1}{v^{2}}+\frac{\lambda R}{\sqrt{\langle z, z\rangle}}\right) z-\left(\frac{1}{v_{0}^{2}}+\frac{\lambda(0) R\left(v_{0}\right)}{\sqrt{\langle w, w\rangle}}\right) w-\int_{0}^{t} \Gamma(x)\left[z,\left(\frac{1}{v^{2}}+\frac{\lambda R}{\sqrt{\langle z, z\rangle}}\right) z\right]+\dot{\lambda} D U(x) d \tau, \\
& F_{2}(z, x, w)=x-\int_{0}^{t} z d \tau .
\end{aligned}
$$


Clearly, if $(z, x, w)$ belongs to $F^{-1}(0,0)$, then the couple $(x(s), v(s))$ satisfies equation (15), besides obviously equation (5) (see (91)), and then it is a critical point for the functional (3) such that $v(0)=v_{0}, \dot{x}(0)=w$. Moreover, since

$$
\dot{\lambda}=R^{\prime}(v) \frac{\sqrt{\langle z, z\rangle}}{v} \lambda-\frac{\langle z, z\rangle}{v^{4}},
$$

using (90) and some easy estimates on the expression $\frac{\lambda}{\sqrt{\langle z, z\rangle}}$, it is possible to see that $F$ is $\mathcal{C}^{1}$ and $F(0,0,0)$ $=(0,0)$. Note that we also use the fact that

$$
v(s) \underset{(x, z) \rightarrow(0,0)}{\longrightarrow} v_{0}>0
$$

To study the dependence of $(x, z)$ on $w$, for $w$ sufficiently small, we will prove that the Jacobian

$$
\frac{\partial F}{\partial(x, z)}(0,0,0): \mathcal{C}^{0}\left([0,1], \mathbb{R}^{2 n}\right) \rightarrow \mathcal{C}^{0}\left([0,1], \mathbb{R}^{2 n}\right)
$$

is invertible. For this aim, let us make a preliminary consideration. We have said that, in (93), $v$ must be seen as a function of the couple $(z(s), x(s))$. Formally differentiating the constraint equation (91) in the direction $(z, x)$, we obtain

$$
V+\frac{1}{v} \int_{0}^{s} R^{\prime}(v) \sqrt{\langle z, z\rangle} V d \tau+\left[\frac{1}{v} \int_{0}^{s} R(v) \frac{\langle z, \zeta\rangle}{\sqrt{\langle z, z\rangle}} d \tau+\langle D U(x), \xi\rangle\right]=0
$$

where

$$
V=d v(z, x)[\zeta, \xi]
$$

and observing that the expression in square parentheses in (97) is uniformly bounded (see also (95)), from Gronwall's lemma we get

$$
\|V\|_{\infty} \leq \nu
$$

for some constant $\nu$, and hence $v$ is uniformly Lipschitz continuous with respect to $(z, x)$. Let us define the following quantity:

$$
\begin{aligned}
\Omega(z, x, w)[\zeta, \xi]=d\left[\left(\frac{1}{v^{2}}+\frac{\lambda R}{\sqrt{\langle z, z\rangle}}\right) z\right][\zeta, \xi]= & -\frac{2}{v^{3}} V z+\frac{R z}{\sqrt{\langle z, z\rangle}} d \lambda(z, x)[\zeta, \xi]+R^{\prime}(v) V \frac{z \lambda}{\sqrt{\langle z, z\rangle}} \\
& +\frac{\lambda R}{\sqrt{\langle z, z\rangle}} \frac{\langle z, \zeta\rangle}{\langle z, z\rangle} z+\left(\frac{1}{v^{2}}+\frac{\lambda R}{\sqrt{\langle z, z\rangle}}\right) \zeta
\end{aligned}
$$


then we have (see (94))

$$
\begin{aligned}
\frac{\partial F_{1}}{\partial z}(z, x, w)[\zeta]+\frac{\partial F_{1}}{\partial x}(z, x, w)[\xi]= & \Omega(z, x, v)[\zeta, \xi]+\left\{\int_{0}^{t} d \Gamma(x)[\xi]\left[z,\left(\frac{1}{v^{2}}+\frac{\lambda R}{\sqrt{\langle z, z\rangle}}\right) z\right]\right. \\
& +\Gamma(x)\left[\zeta,\left(\frac{1}{v^{2}}+\frac{\lambda R}{\sqrt{\langle z, z\rangle}}\right) z\right]+\Gamma(x)[z, \Omega]+d \dot{\lambda}(z, x)[\zeta, \xi] D U(x) \\
& +\dot{\lambda}\langle D U(x), \xi\rangle d \tau\}+\frac{R\left(v_{0}\right) d \lambda(0)[\zeta, \xi]}{\sqrt{\langle w, w\rangle}} w .
\end{aligned}
$$

Using some elementary estimates it is easily seen that, evaluating $(101)$ at $(z, x, w)=(0,0,0)$, we get

$$
\frac{\partial F_{1}}{\partial z}(z, x, w)[\zeta]+\frac{\partial F_{1}}{\partial x}(z, x, w)[\xi]=\frac{1}{v_{0}^{2}} \zeta
$$

whereas it is immediate to realize that

$$
\frac{\partial F_{2}}{\partial z}(z, x, w)[\zeta]+\frac{\partial F_{2}}{\partial x}(z, x, w)[\xi]=\xi-\int_{0}^{t} \zeta d \tau .
$$

Now the map

$$
\frac{\partial F}{\partial(x, z)}(0,0,0)[\zeta, \xi]=\left(\frac{1}{v_{0}^{2}} \zeta, \xi-\int_{0}^{t} \zeta d \tau\right)
$$

is invertible, with inverse given by

$$
\left(\frac{\partial F}{\partial(x, z)}(0,0,0)\right)^{-1}[\zeta, \xi]=\left(v_{0}^{2} \zeta, \xi+v_{0}^{2} \int_{0}^{t} \zeta d \tau\right) .
$$

Then, by the Implicit Function Theorem, for $w$ sufficiently small there is a $\mathcal{C}^{1}$ map

$$
w \mapsto\left(z_{w}, x_{w}\right)
$$

such that

$$
F\left(z_{w}, x_{w}, w\right)=0 .
$$

Note that the differential of this map at $w=0$ is easily computed:

$$
-\left(\frac{\partial F}{\partial(x, z)}(0,0,0)\right)^{-1}\left[\frac{\partial F}{\partial v}(0,0,0)[\omega]\right]=(\omega, \omega \cdot t) .
$$

This shows that, for every $w$ in a neighborhood of 0 of $T_{p} \mathcal{M}$, there exists a unique curve $\left(x_{w}, v_{w}\right)$ satisfying (5) and (15), and such that $x_{w}(0)=p, \dot{x}_{w}(0)=w, v_{w}(0)=v_{0}$. Moreover (106) shows that the exponential map $\mathfrak{e x p}_{p}: T_{p} \mathcal{M} \rightarrow \mathcal{M}$, defined as

$$
\mathfrak{e x p}_{p}(w)=x_{w}(1),
$$

is such that its differential is the identity map on $T_{p} \mathcal{M}$, and then $\mathfrak{e x p}_{p}$ is a local diffeomorphism. Thus, if $q$ is sufficiently close to $p$, there exists a unique $\mathcal{C}^{2}$ solution $(x, v)$ of $(5)$ and (15) that joins $p$ and $q$ with initial velocity $v_{0}$. We have therefore proven the following: 
Proposition 3.1. For every $p \in \mathcal{M}, v_{0} \in \mathbb{R}_{+}$, there exists a neighborhood of $p$ in $\mathcal{M}$ such that for every $q$ in this neighborhood there exists a unique $\mathcal{C}^{2}$ minimizer of (3) (namely a brachistocrone curve) from $p$ to $q$ with initial velocity $v_{0}$.

\section{EXAmples}

1. Let us consider the case when the frictional force of the resisting medium is linear,

$$
R(v)=\alpha v, \quad \alpha \neq 0
$$

Such an $R(v)$ verifies assumption (10); in this case the scalar function $P(s)(16)$ is

$$
P(s)=C^{2} v(s) e^{\alpha C s} \int_{s}^{1} \frac{1}{v(\sigma) e^{\alpha C \sigma}} d \sigma,
$$

where

$$
\sqrt{\langle\dot{x}(s), \dot{x}(s)\rangle}=C v(s)
$$

and equation (15) becomes

$$
\begin{aligned}
& \left(\frac{1}{v^{2}}+\alpha C e^{\alpha C s} \int_{s}^{1} \frac{1}{v^{2} e^{\alpha C \sigma}} d \sigma\right) D_{s} \dot{x}+ \\
& -\left(2 \frac{\dot{v}}{v}+\frac{\alpha C}{v^{2}}-\alpha^{2} C^{2} e^{\alpha C s} \int_{0}^{1} \frac{1}{v^{2} e^{\alpha C \sigma}} d \sigma\right) \dot{x}+\left(\alpha C^{3} e^{\alpha C s} \int_{s}^{1} \frac{1}{v^{2} e^{\alpha C \sigma}} d \sigma-\frac{C^{2}}{v^{2}}\right) D U(x)=0 .
\end{aligned}
$$

2. We can observe that the above equation becomes a true differential equation in the frictionless case, that is when $\alpha=0$. Indeed, in this case (110) becomes

$$
D_{s}\left(\frac{\dot{x}}{v^{2}}\right)=\frac{C^{2}}{v^{2}} D U(x) .
$$

We also observe that the constraint equation (11) now gives

$$
v^{2}=2(E-U(x)),
$$

and then we recover the equation of the brachistochrone in a conservative force field:

$$
D_{s} \dot{x}=C^{2} D U(x)-\frac{\langle D U(x), \dot{x}\rangle}{2(E-U(x))} \dot{x} .
$$

Note that in absence of potential (113) is the equation of the geodesics in $\mathcal{M}$.

3. As a particular case of Example 2, let us see the solution of the classical problem.

In this case $\mathcal{M}=\mathbb{R}^{2}, U(x, y)=y, R(v)=0$. Let $p=O$, the axes origin, and $q=(\bar{x}, \bar{y})$. Then (113) yields

$$
\frac{d}{d s}\left(\frac{\dot{x}}{v^{2}}\right)=0, \quad \frac{d}{d s}\left(\frac{\dot{y}}{v^{2}}\right)=\frac{C^{2}}{v^{2}} .
$$

Using these equations together with (14) and (112) we come to the following equations:

$$
\begin{aligned}
& \dot{x}(t)=a(E-y(t)), \\
& \ddot{y}(t)+a^{2} y(t)+\left(C^{2}-a^{2} E\right)=0,
\end{aligned}
$$


where $E=\frac{1}{2} v_{0}^{2}$, and $a, C$ are constants to determine. With some calculation we find the solution

$$
\begin{aligned}
& x(t)=\frac{C^{2}}{a^{2}}\left(a\left(t+t_{0}\right)-\sin \left(a\left(t+t_{0}\right)\right)\right)+\frac{C^{2}}{a^{2}}\left(\sin a t_{0}-a t_{0}\right), \\
& y(t)=\frac{C^{2}}{a^{2}}\left(\cos \left(a\left(t+t_{0}\right)\right)-1\right)+E, \\
& v(t)=2 \frac{C}{a} \sin \left(\frac{a\left(t+t_{0}\right)}{2}\right),
\end{aligned}
$$

where

$$
\cos \left(a t_{0}\right)=1-E \frac{a^{2}}{C^{2}}
$$

and $a, C$ must be determined using the conditions

$$
x(1)=\bar{x}, \quad y(1)=\bar{y} .
$$

\section{REFERENCES}

[1] H. Brezis, Analyse fonctionnelle. Masson, Paris (1983).

[2] F. Giannoni, P. Piccione and J.A. Verderesi, An approach to the relativistic brachistochrone problem by sub-Riemannian geometry. J. Math. Phys. 38 (1997) 6367-6381.

[3] F. Giannoni and P. Piccione, An existence theory for relativistic brachistochrones in stationary space-times. J. Math. Phys. 39 (1998) 6137-6152.

[4] J. Nash, The embedding problem for Riemannian manifolds. Ann. Math. 63 (1956) 20-63.

[5] L.A. Pars, An Introduction to the Calculus of Variations. Heinemann, London (1962).

[6] V. Perlick, The brachistochrone problem in a stationary space-time. J. Math. Phys. 32 (1991) 3148-3157. 